\title{
Test of the Electron-Neutron Detector Array (ENDA) in Laboratory
}

\author{
Fan Yang, ${ }^{a}$ Shu-Wang Cui, ${ }^{a}, *$ Tian-Lu Chen, ${ }^{b, c}$ Dangzengluobu, ${ }^{b, c}$ Denis Kuleshov, ${ }^{d}$ \\ Mao-Yuan Liu, ${ }^{b, c}$ Bing-Bing Li, ${ }^{a}$ Ye Liu, ${ }^{e}$ Xin-Hua Ma, ${ }^{f, g}$ Cong Shi, ${ }^{a}$ Oleg \\ Shchegolev, ${ }^{d}$ Yuri Stenkin, ${ }^{d}$ Vladimir Stepanov, ${ }^{d}$ Dixuan Xiao ${ }^{b, c}$ and Liang-wei \\ Zhang $^{a}$ \\ ${ }^{a}$ The College of Physics, Hebei Normal University, \\ 050024,Shijiazhuang, China \\ ${ }^{b}$ Science School, Tibet University, \\ 850000,Lhasa, China \\ ${ }^{c}$ Key Laboratory of Comic Rays, Tibet University, Ministry of Education, \\ 850000, Lhasa, China \\ ${ }^{d}$ Institute for Nuclear Research, Russian Academy of Science, \\ 117312,Moscow, Russia \\ ${ }^{e}$ School of Management Science and Engineering, Hebei University of Economics and Business, \\ 050024,Shijiazhuang, China \\ ${ }^{f}$ Key Laboratory of Particle Astrophysics, Institute of High Energy Physics, \\ 100049,Bejing, China \\ ${ }^{g}$ TIANFU Cosmic Ray Research Center, \\ 610000,Chengdu, China \\ E-mail: cuisw@hebtu.edu.cn, yf18233557750@163.com
}

Abstract: The measurement of thermal neutrons generated by cosmic ray extensive air showers (EAS) on the Earth's surface provides a new method for studying the composition and energy spectrum of cosmic rays at the so-called "knee" region. The electron-neutron detector (ENdetector) utilizing a new type scintillator based on a compound alloy of $\mathrm{ZnS}(\mathrm{Ag})$ and $\mathrm{B}_{2} \mathrm{O}_{3}$ with natural boron is developed. At present 2 clusters (each consists of 16 detectors) are kept at the laboratory in Hebei Normal University (HNU). The performance test of the EN-detector mainly focuses on role of the cone, and the detector noise level.

$37^{\text {th }}$ International Cosmic Ray Conference (ICRC 2021)

July 12th-23rd, 2021

Online-Berlin, Germany

\footnotetext{
${ }^{*}$ Presenter
} 


\section{Introduction}

The cosmic ray energy spectrum basically obeys a power law. But around $4 \times 10^{15} \mathrm{eV}$, at the so-called "knee" region, spectrum index decreases into -3.1 from -2.7. The mechanism of knee region is supposed to relate to the origin, acceleration, and propagation of cosmic rays. However, so far, many ground-based experiments don't give consistent results. Such as KASCADE[1] and Tibet-AS $\gamma$ [2], although they confirmed the existence of knee region, the results are quite different, even contradictory. The exact power law index, proportion of components, and location of knee region are still ambiguous. Hadronic components are "skeleton" of EAS[3] and possess information concerning energy and properties of primary particles. Hadrons will react with surround materials (such as soil, building, and detectors materials) of the detectors and produce evaporation neutrons. Those evaporation neutrons will be moderated by matter in surrounding environment, becoming thermal neutrons. The total number of thermal neutrons is usually two or three order of magnitude more than that of hadrons[4]. Furthermore, quantity of neutrons is sensitive to primary particles: thermal neutrons generated by light nuclei (such as proton) are one order more than heavy nuclei (such as iron)[5]. So, the electron-neutron detector (EN-detector) have better performance-cost radio than hadron calorimeters in the scope of compositions identification [6].

At present 2 clusters(32 detectors) are kept at the laboratory in Hebei Normal University (HNU). The performance test of the EN-detector includes the relative gain calibration, the neutron detection efficiency, comparision of detection dfficiencies between deficiencies types of the cone. The test will measure noise level of detector and understand the role of the cone.

\section{Experimental Setup}

The structure of the EN-detector is illustrated in figure.1. The main component of the detector is a black polythylene (PE) barrel, with a scintillator at the bottom, and a 4-inch potomulitiplier (PMT) at the top. A reflector cone is used to collect scintillation photons from the scintillator to PMT. The EN-detector is based on a novel type scintillator, which is a granulated alloy of inorganic $\mathrm{ZnS}(\mathrm{Ag})$ scintillator dopped with natural $\mathrm{B}_{2} \mathrm{O}_{3}$ in which the percentage ${ }^{10} \mathrm{~B}$ isotope is about $20 \%$. One ${ }^{10} \mathrm{~B}$ nucleus captures one thermal neutron via the reaction:

$$
\begin{aligned}
{ }^{10} \mathrm{~B}+n & \rightarrow^{7} \mathrm{Li}^{*}(0.84 \mathrm{MeV})+\alpha(1.47 \mathrm{MeV}) \rightarrow^{7} \mathrm{Li}+\alpha+\gamma(0.478 \mathrm{MeV}), Q=2.310 \mathrm{MeV}, 93.7 \% \\
& \rightarrow^{7} \mathrm{Li}(1.01 \mathrm{MeV})+\alpha(1.78 \mathrm{MeV}), Q=2.790 \mathrm{MeV}, 6.3 \%
\end{aligned}
$$

At present 2 clusters (32 detectors) are deployed at the laboratory, shown in figure.2. Because of small laboratory space, the distance between every two adjacent detectors is about $1 \mathrm{~m}$. The array was arranged in two layers. One cluster on the top called IP12 according to the IP address of the connected FADC, another on the below called IP11. Each cluster has its own FADC. These FADCs transmit data to PC by the White Rabbit Switch (WRS). To quantify the role of the cone, we took out the cone and compared counting rate. We took out the scintillator at detector No.5 in the IP11 cluster to test detector noise level. Detector No.10 in the IP11 array was used to study on role of the cone. Detector No.6 in the IP12 cluster is used as a standard detector. Test process is shown in Table 1. In period. 1 the two detectors normal operation and in period. 2 we removed the scintillator of detector No.5 and the cone of detector No.10. 


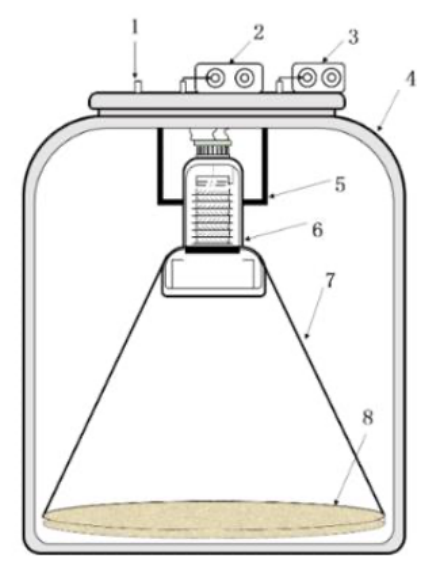

Figure 1: Schematic of the EN-detector. 1- HV input port, 2- d8 preamplifier(DIU), 3- d5 preamplifier (UI), 4- black tank, 5- PMT fixed holder, 6-PMT, 7- light collecting cone, 8- scintillator

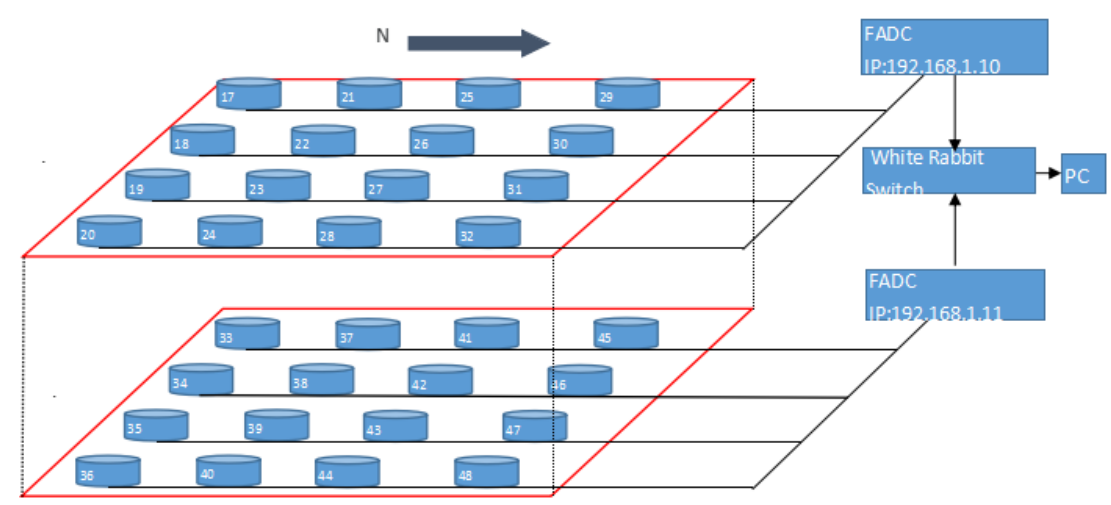

Figure 2: Configuration of 2 clusters (32 detectors) at the laboratory in Hebei Normal University.

\section{Data analysis and results}

The No.6 detector is regarded as a standard detector, which is stable in these periods, as shown in figure.3. It indicates the environmental condition of it is stable. After two days of normal operation, the scintillator of detector No.5 and the cone of detector No.10 in cluster IP11 are removed.

Percentage of noise in signal (neutrons and charged particles) counts can be obtained by removing the scintillator. From figure. 4 and Table 2, it is obtained that percentage of noise in neutrons and charged particles is $12.9 \%$ and $86.8 \%$ respectively. Percentage of light collection by the cone in signal counts can be obtained by removing the cone. From figure. 5 and Table 2, it is obtained that percentage of light collection by the cone in neutrons and charged particles is $1-25.8 \%=74.2 \%$ and $1-84.5 \%=15.5 \%$ respectively.

\section{Summary}

During test of EN-detectors in laboratory, percentage of noise in counting rate of neutrons and charged particles is obtained, and function of the reflector is studied. In the near future, we will 
Table 1: Test process

\begin{tabular}{ccc}
\hline Detector number & Data period & Process \\
\hline No.06 in IP12 & $01 / 28 / 2021-01 / 29 / 2021$ & Normal operation \\
& $02 / 01 / 2021-02 / 09 / 2021$ & Normal operation \\
No.05 in IP11 & $01 / 28 / 2021-01 / 29 / 2021($ period No.1) & Normal operation \\
& $02 / 01 / 2021-02 / 09 / 2021($ period No.2) & Remove scintillator \\
No.10 in IP11 & $01 / 28 / 2021-01 / 29 / 2021($ period No.1) & Normal operation \\
& $02 / 01 / 2021-02 / 09 / 2021($ period No.2) & Remove the cone \\
\hline
\end{tabular}

Table 2: Test result

\begin{tabular}{ccccc}
\hline $\begin{array}{c}\text { Detector No. } \\
\text { in IP11 }\end{array}$ & signal type & $\begin{array}{c}\text { average value/hour } \\
\text { (period No.1 })\end{array}$ & $\begin{array}{c}\text { average value/hour } \\
\text { (period No.2) }\end{array}$ & $\begin{array}{c}\text { ratio of period } \\
\text { No.2 to No.1 }\end{array}$ \\
\hline \multirow{2}{*}{05} & neutron & 184.1 & 23.5 & $12.9 \%$ \\
& charged & 1170.6 & 1017.2 & $86.8 \%$ \\
10 & neutron & 146.5 & 37.7 & $25.8 \%$ \\
& charged & 912.9 & 771.4 & $84.5 \%$ \\
\hline
\end{tabular}

keep on test to study for optimization of performance of the detectors.

\section{Acknowledgments}

This work was supported in China by National Natural Science Foundation of China (NSFC) (No. U1831129, U2031103, 11905043, U1831208, 11963004, 12047575), Everest Discipline Construction Plan of Tibet University and by the Science and Technology Department of Sichuan Province (grant number 2021YFSY0031). 

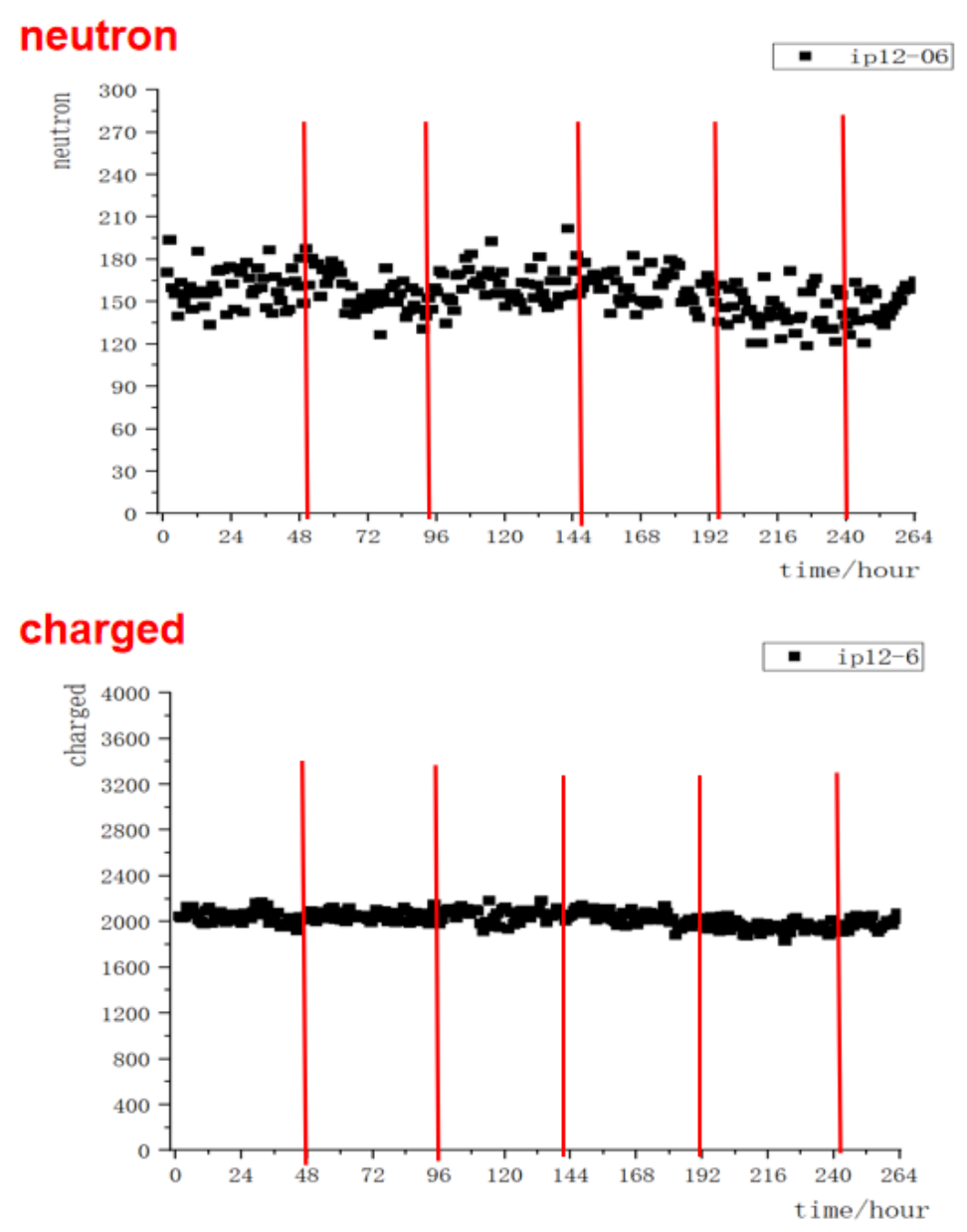

Figure 3: Variation of counting rate. detector No.06 in cluster IP12

\section{References}

[1] Antoni T, Apel W D, Badea A F, et al. KASCADE measurements of energy spectra for elemental groups of cosmic rays: Results and open problems[J]. Astroparticle physics, 2005, 24(1-2): 1-25.

[2] Amenomori M, Bi X J, Chen D, et al. The all-particle spectrum of primary cosmic rays in the wide energy range from $10^{14}$ to $10^{17} \mathrm{eV}$ observed with the Tibet-III air-shower array[J]. The Astrophysical Journal, 2008, 678(2): 1165.

[3] Zatsepin G T. Nuclear-Cascade Process and Its Part in the Development of Extensive Atmospheric Showers[C]. Doklady Akad. Nauk. SSSR. 1949, 67, p. 993.

[4] Stenkin Y V, Alekseenko V V, Gromushkin D M, et al. Thermal neutron flux produced by EAS at various altitudes[J]. Chinese physics C, 2013, 37(1): 015001.

[5] Stenkin Y V, Valdes-Galicia J F. On the neutron bursts origin[J]. Modern Physics Letters A, 2002, 17(26): 1745-1751. 


\section{neutron Remove scintillator}

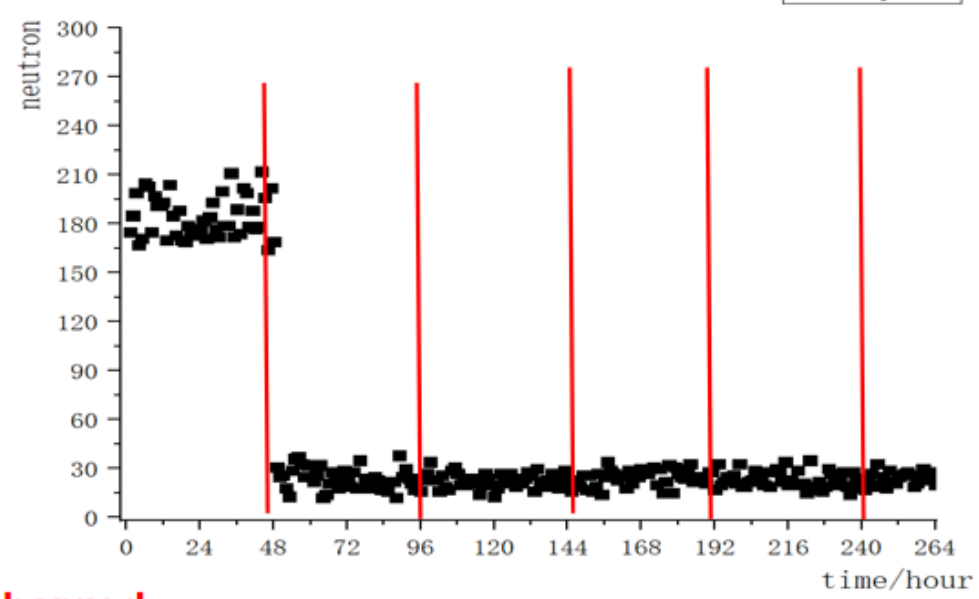

\section{charged}

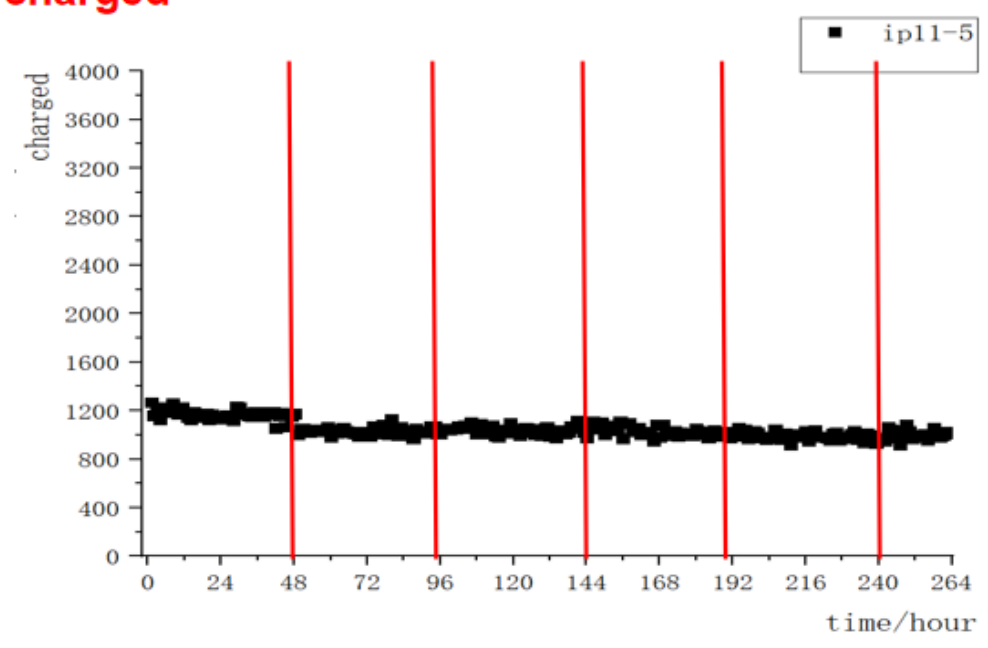

Figure 4: Variation of counting rate. detector No.05 in cluster IP11.

[6] Stenkin Y, Alekseenko V, Cai Z, et al. Response of the environmental thermal neutron flux to earthquakes[J]. Journal of environmental radioactivity, 2019, 208: 105981. 

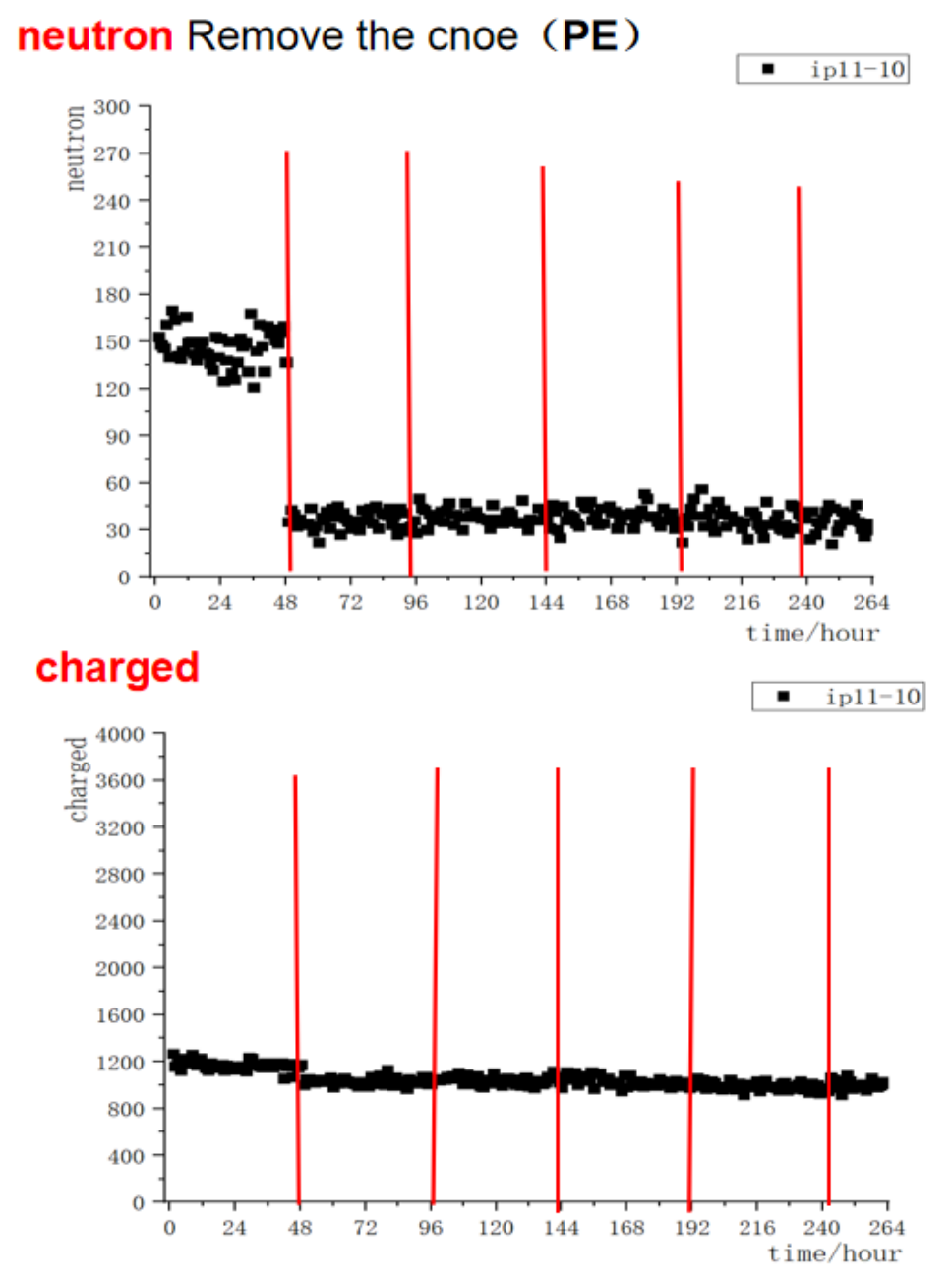

Figure 5: Variation of counting rate. detector No.10 in cluster IP11. 